\title{
A Novel Motion Estimation Approach for Video Coding: Direct Path for Block Matching (DPBM)
}

\author{
Mona A. M. Fouad ${ }^{1}$ and Ahmed Mokhtar A. Mansour ${ }^{2}$ \\ ${ }^{1}$ National Telecommunication Institute, Cairo, Egypt \\ ${ }^{2}$ Nile Innovations, Cairo, Egypt \\ Imfouad@nti.sci.eg, 2ahmedmokhtar_adu@yahoo.com
}

\begin{abstract}
The paper proposes a new approach to detect the matched Macroblock in the reference frame for motion estimation in video codec. According to the fact that the content of a MB is correlated and also the surrounding within a small area, the algorithm detects the shortest direct path to the matched Macroblock. This will be performed by tracking the gradual decrease of the differences of the compared Macroblocks. We start from the corresponding initial position at the predicted frame in the reference frame according to a novel and simple criteria. The proposed algorithm is developed and evaluated using benchmark video sequences of various types, achieving computation reduction up to $98 \%$.
\end{abstract}

Keywords: Abrupt transitions, best matched MacroBlock (MB), Motion Compensation $(M C)$, video coding

\section{Introduction}

Motion compensation is essential for encoding video sequences such as the ISO/IEC MPEG-1, -2, -3 and the ITU-T H.261, 262, 263 as well as the H.264/AVC and the H.265/HEVC. The motion is compensated by encoding the difference between the target frame and a previously encoded frame(s). This is done by partitioning frames into non-overlapped MBs, and then processing each of them individually. The considered MB is compared to its corresponding position in the reference frame and its neighbors within small area (search window), searching for the best matched MB. The comparison is performed using the Absolute Mean Difference (AMD). If the best match is found, then the difference between the target MB and the best matched MB is encoded and transmitted in addition to the positions of the matched MBs that are stored in Motion Vectors (MV). If not, the considered MB is intra-encoded [1].

The search process is the key player of the motion compensation process. Several algorithms are explored to efficiently detect the best matched MB. The most famous ones are the Exhaustive Search (ES), the Three Step Search (TSS), the New Three Step Search (NTSS), the Simple and Efficient Search (SES), the Four Step Search (4SS), the Diamond Search (DS), and the Adaptive Rood Pattern Search (ARPS). Several works are developed to review and compare some or all these algorithms [2]-[4]. Other works are conducted to enhance these algorithms, such as those in [5]-[8].

In this paper, the direct path to the best matched $\mathrm{MB}$ is discovered based on a novel and simple criteria. The path is detected if the continuous degradation of the AMD of the corresponding $\mathrm{MB}$ in the reference frame and its four neighbors is tracked. The DPBM algorithm ensures detection of the shortest path to the best matched MB. Computation reduction is achieved by reducing the number of MBs to

Received (November 28, 2017), Review Result (January 2, 2018), Accepted (January 5, 2018) 
be examined, in the reference frame. The proposed DPBM algorithm decides, very early, whether to continue looking for the best match, or to exit with/without detecting it. If the termination is performed with the detected best match, then the shortest path is discovered. If the termination is performed without detecting the best match, it means that the continuation of the search process will not reach to it, and the best match does not exist within the considered search window. In this case the MB will be intra-encoded.

Concerning the case of abrupt transition, where each of the reference frame and the target frame belongs to different shots (or scenes). Then all MBs of the target frame are candidates to be intra-coded. Using DPBM, the computations are highly reduced from the very early, while other algorithms waste computation time and at last will take the same decision.

The detailed steps of the proposed algorithm are illustrated in Section 2. Sections 3 and 4 demonstrate the conducted experiments and some practical examples, respectively. Finally, the presented work is summarized in Section 5.

\section{The DPBM Algorithm}

The DPBM algorithm searches for the best matched MB in the reference frame, starting at the corresponding position of the target MB. The search process is applied within a window of size $(2 \mathrm{p}+1)^{\wedge} 2$, as shown in Figure 1.

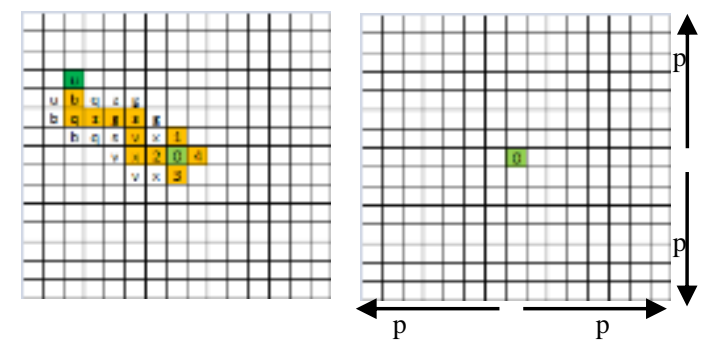

\section{Figure 1. The Considered MBs in the Target Frame (right) and its Corresponding in the Reference Frame (left) within a 15x15 Search Window $(p=7)$}

The flowchart shown in Figure 2 summarizes the DPBM procedure that searches for the best matched MB in the target frame by estimating the AMD between the considered $\mathrm{MB}$ in the target frame and the candidate MBs in the reference frame.

The procedure comprises four conditions; two of them terminate the procedure promptly. First, if the difference is less than certain predefined threshold (it is zero in our case study), then the procedure is terminated and the best match is detected at the initial position in the reference frame. Second, the AMDs of the target MB are examined against their corresponding of the reference MB; (AMD0) and its four neighbors (up, down, right, left) in the reference frame (AMD1, AMD2, AMD3, AMD4). If all the four AMDs of the four neighbors are greater than the AMD0, then the procedure is terminated and the target MB will be intra-coded. Third, if there is one (or more) AMD of the four neighbors is smaller or equal to the AMD0, then the greatest of these values (if more than one neighbor is smaller than AMD0) is picked up to be the initial position for the next iteration. Fourth, if the AMD0 is greater than all its neighbors during any of the successive iterations, then this is the end of the tracked path and the current position of the MB in the reference frame is the best match. Thus, the procedure is repeated until one of the termination conditions is satisfied. 


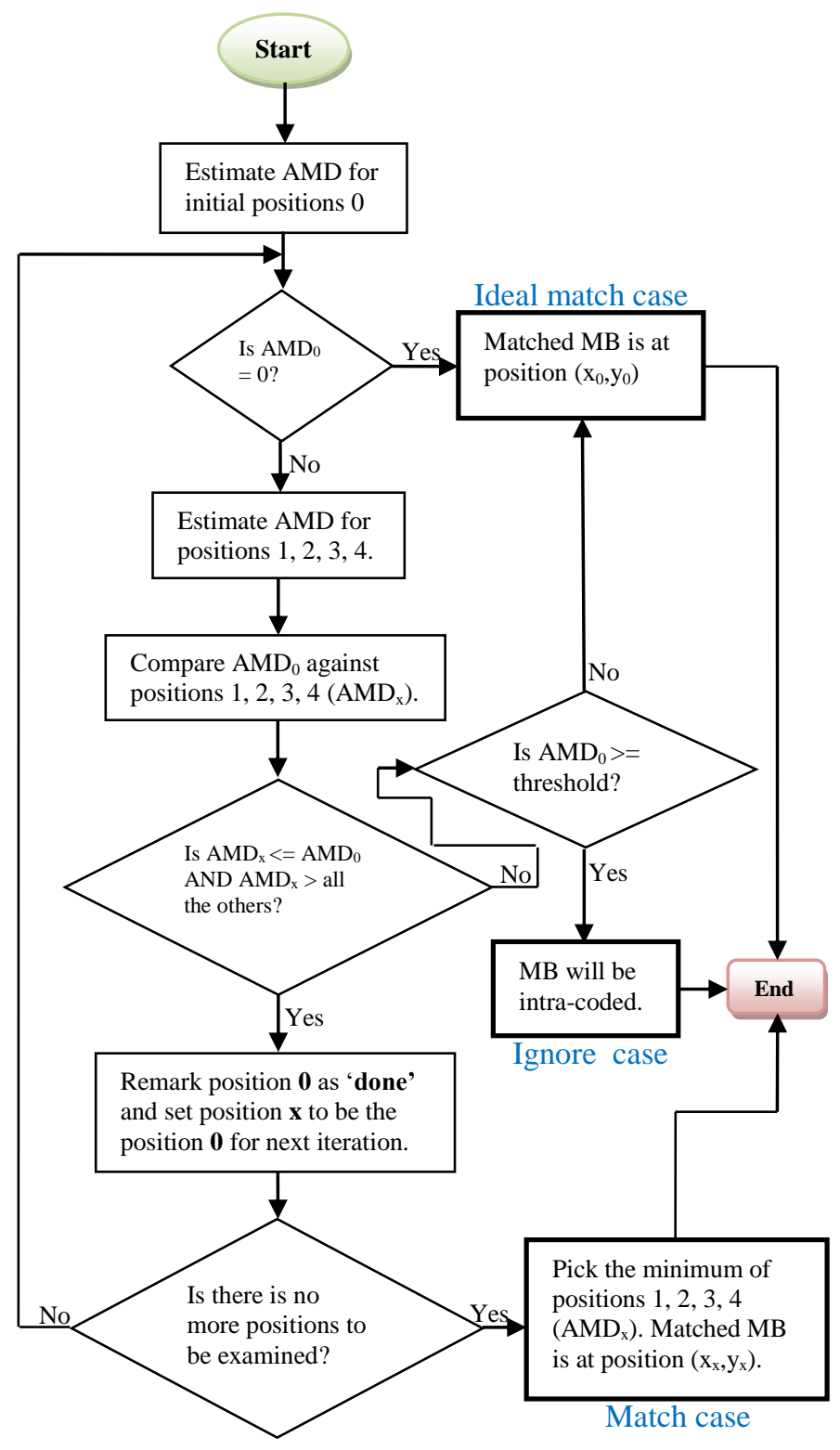

Figure 2. The Flowchart of the DPBM Algorithm

\section{Experiments and Results}

The DPBM algorithm reduces, significantly, the computations needed to encode predicted frames through: 1) promptly terminating the search process in the very early, deciding that the MB should be intra-encoded, 2) discovering the shortest path by promptly terminates the search process once the best match is detected and there is no further relevant MBs could be candidates for the best matching.

No preprocessing or additional processing is performed to detect the best relevant matched MB. Only the AMD is estimated to compare the difference between the corresponding MBs. The proposed DPBM algorithm is evaluated by comparing it to six well known algorithms; TSS, SESTSS, NTSS, 4SS, DS, and ARPS, concerning the computation reduction to detect the best matched MB.

The DPBM algorithm is applied to a test-bench composed of five different types of video clips, 'news', 'wedding', 'soccer', 'racing, 'horse racing'. Six hundred frames of each clip are randomly selected for evaluation, with frame size of 
352x352. Each frame is partitioned into non-overlapped 16x16 MBs. Three experiments are explored at which the target frame is apart from the reference frame by 1,2 , and 4 frames.

The 'Soccer' movie has common background and common sport wears. The 'Wedding' movie has common background and slow motion of objects. The 'Horse Racing' movie has common background and very fast motion of objects (horses). The 'News' movie has shadows and unconditional lighting effect as well as the sudden change of the News bar. The 'Racing' movie has very fast moving of objects (racers) and rapid changes of the camera direction. Sample frames of the test-bench video clips are shown in Figure 3.

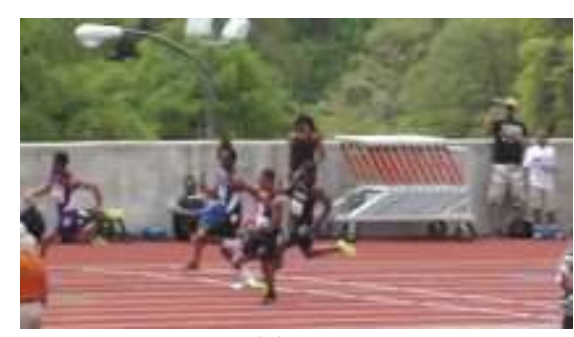

(a)

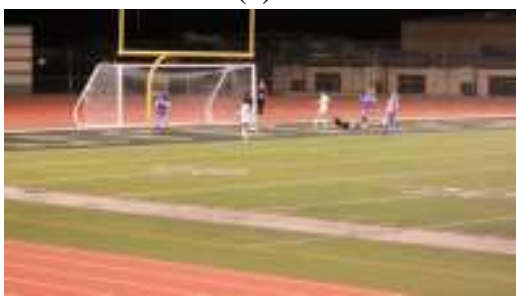

(c)

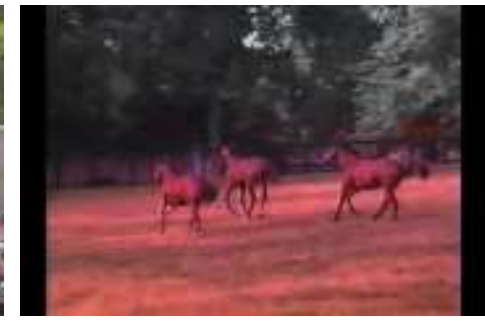

(b)

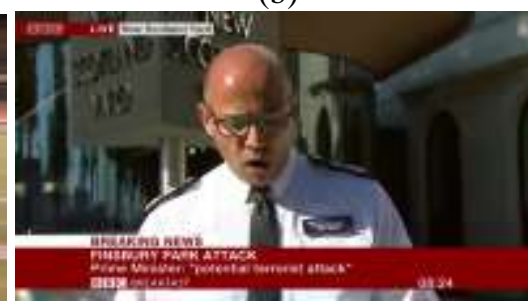

(d)

Figure 3. Samples of the Test-Bench Video Clips: (a) 'Racing', (b) 'Horse Racing', (c) 'Soccer', and (d) 'News'

The MATLAB implementation of the seven algorithms, namely; ES, TSS, SESTSS, NTSS, 4SS, DS, and ARPS, performed by Aloh Barjatya is used [2]. The implementation is developed and adopted to be applied for the test-bench video clips used for evaluation. The DPBM algorithm is also implemented and the results are compared to the output of the six algorithms, excluding the exhaustive algorithm as it is the reference for all other algorithms. The conducted experiments suppose that the search process is performed looking for the minimum AMD over the whole search window and the comparison is applied for typical equality.

The overall results of the bench mark videos are summarized in Table 1, showing that the DPBM is comparable, in its trivial setup. Generally, the DPBM algorithm outperforms other algorithms concerning the number of detections, and the computation reduction percentage for the 'Wedding' and 'Soccer' video clips. With respect to the 'Horse Racing' clip, the detection percentage is comparable when the reference frame is apart by one or two frames. When the reference frames are apart by four frames the detection percentage is reduced due to the fast moving of the foreground objects (horses). The DPBM algorithm results, in case of the 'News' clip, are also comparable concerning the detection percentage, and it is the best for the computation reduction. The DPBM algorithm results are comparable in case of the 'Racing' clip, concerning the detection percentage, but are the lowest among other movies. This is due to the fast movement of racers and the rapid change of the cameras' point of views. 
Table 1. The Average Number of Detections and Computations of the DPBM and the Six Search Algorithms at steps 1, 2, and 4

\begin{tabular}{|c|c|c|c|c|c|c|c|c|}
\hline & & DPBM & TSS & SESTSS & NTSS & 4SS & DS & ARPS \\
\hline \multirow[b]{4}{*}{$\infty$} & \multirow{4}{*}{ Step 1} & 456 & 475 & 465 & 452 & 468 & 465 & 469 \\
\hline & & $94 \%$ & $98 \%$ & $96 \%$ & $93 \%$ & $97 \%$ & $96 \%$ & $97 \%$ \\
\hline & & 3459 & 6633 & 11362 & 6947 & 8998 & 7925 & 3869 \\
\hline & & $97 \%$ & $93 \%$ & $89 \%$ & $93 \%$ & $91 \%$ & $92 \%$ & $96 \%$ \\
\hline 0 & \multirow{4}{*}{ Step 2} & 454 & 444 & 430 & 365 & 436 & 426 & 439 \\
\hline$\Omega$ & & $94 \%$ & $92 \%$ & $89 \%$ & $75 \%$ & $90 \%$ & $88 \%$ & $91 \%$ \\
\hline 2 & & 3491 & 9424 & 11646 & 6872 & 11436 & 9292 & 5454 \\
\hline (x) & & $97 \%$ & $91 \%$ & $88 \%$ & $93 \%$ & $89 \%$ & $91 \%$ & $95 \%$ \\
\hline \multirow[t]{4}{*}{$\approx$} & \multirow{4}{*}{ Step 4} & 445 & 444 & 425 & 357 & 436 & 423 & 438 \\
\hline & & $92 \%$ & $92 \%$ & $88 \%$ & $74 \%$ & $90 \%$ & $87 \%$ & $91 \%$ \\
\hline & & 4753 & 9830 & 12153 & 6898 & 11496 & 9604 & 6056 \\
\hline & & $95 \%$ & $90 \%$ & $88 \%$ & $93 \%$ & $88 \%$ & $90 \%$ & $94 \%$ \\
\hline \multirow[b]{3}{*}{$\sum$} & \multirow{4}{*}{ Step 1} & 483 & 484 & 484 & 483 & 484 & 484 & 484 \\
\hline & & $100 \%$ & $100 \%$ & $100 \%$ & $100 \%$ & $100 \%$ & $100 \%$ & $100 \%$ \\
\hline & & 2424 & 5474 & 7748 & 6933 & 11320 & 7230 & 2437 \\
\hline \multirow{4}{*}{$\begin{array}{l}x \\
\theta \\
\theta\end{array}$} & & $98 \%$ & $95 \%$ & $92 \%$ & $93 \%$ & $89 \%$ & $93 \%$ & $98 \%$ \\
\hline & \multirow{4}{*}{ Step 2} & 473 & 459 & 464 & 435 & 465 & 457 & 459 \\
\hline & & $98 \%$ & $95 \%$ & $96 \%$ & $90 \%$ & $96 \%$ & $94 \%$ & $95 \%$ \\
\hline & & 2471 & 6533 & 8814 & 6912 & 11355 & 7810 & 3292 \\
\hline \multirow[t]{5}{*}{$Z \Omega$} & & $98 \%$ & $93 \%$ & $91 \%$ & $93 \%$ & $89 \%$ & $92 \%$ & $97 \%$ \\
\hline & \multirow{4}{*}{ Step 4} & 470 & 455 & 461 & 422 & 461 & 453 & 453 \\
\hline & & $97 \%$ & $94 \%$ & $95 \%$ & $87 \%$ & $95 \%$ & $94 \%$ & $94 \%$ \\
\hline & & 2569 & 6798 & 9097 & 6909 & 11364 & 7959 & 3562 \\
\hline & & $97 \%$ & $93 \%$ & $91 \%$ & $93 \%$ & $89 \%$ & $92 \%$ & $96 \%$ \\
\hline 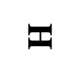 & \multirow{4}{*}{ Step 1} & 479 & 482 & 482 & 480 & 482 & 481 & 478 \\
\hline \multirow{2}{*}{$\begin{array}{l}0 \\
\nabla\end{array}$} & & $99 \%$ & $100 \%$ & $100 \%$ & $99 \%$ & $100 \%$ & $99 \%$ & $99 \%$ \\
\hline & & 2618 & 5637 & 7974 & 6926 & 11322 & 7319 & 2682 \\
\hline $\boldsymbol{n}$ & & $97 \%$ & $94 \%$ & $92 \%$ & $93 \%$ & $89 \%$ & $93 \%$ & $97 \%$ \\
\hline W & \multirow{4}{*}{ Step 2} & 478 & 483 & 484 & 474 & 478 & 481 & 479 \\
\hline \multirow[t]{2}{*}{$\approx$} & & $99 \%$ & $100 \%$ & $100 \%$ & $98 \%$ & $99 \%$ & $99 \%$ & $99 \%$ \\
\hline & & 2591 & 5590 & 7882 & 6942 & 11331 & 7300 & 2564 \\
\hline$\Omega$ & & $97 \%$ & $94 \%$ & $92 \%$ & $93 \%$ & $89 \%$ & $93 \%$ & $97 \%$ \\
\hline- & \multirow{4}{*}{ Step 4} & 362 & 378 & 248 & 353 & 386 & 426 & 403 \\
\hline \multirow{3}{*}{$\begin{array}{l}\mathbf{Z} \\
\Omega\end{array}$} & & $75 \%$ & $78 \%$ & $51 \%$ & $73 \%$ & $80 \%$ & $88 \%$ & $83 \%$ \\
\hline & & 3867 & 11485 & 6881 & 13068 & 10372 & 11249 & 7156 \\
\hline & & $96 \%$ & $88 \%$ & $93 \%$ & $87 \%$ & $90 \%$ & $89 \%$ & $93 \%$ \\
\hline & Step 1 & 429 & 461 & 459 & 429 & 448 & 457 & 457 \\
\hline
\end{tabular}




\begin{tabular}{|c|c|c|c|c|c|c|c|c|}
\hline \multirow{11}{*}{$\begin{array}{l}Z \\
\text { W } \\
\& \\
\infty\end{array}$} & & $89 \%$ & $95 \%$ & $95 \%$ & $89 \%$ & $93 \%$ & $94 \%$ & $94 \%$ \\
\hline & & 5357 & 6614 & 9107 & 6896 & 11347 & 7940 & 3823 \\
\hline & & $95 \%$ & $93 \%$ & $91 \%$ & $93 \%$ & $89 \%$ & $92 \%$ & $96 \%$ \\
\hline & \multirow{4}{*}{ Step 2} & 414 & 450 & 440 & 405 & 433 & 430 & 456 \\
\hline & & $86 \%$ & $93 \%$ & $91 \%$ & $84 \%$ & $89 \%$ & $89 \%$ & $94 \%$ \\
\hline & & 6884 & 7363 & 9899 & 6875 & 11351 & 8361 & 4403 \\
\hline & & $93 \%$ & $93 \%$ & $90 \%$ & $93 \%$ & $89 \%$ & $92 \%$ & $96 \%$ \\
\hline & \multirow{4}{*}{ Step 4} & 381 & 433 & 414 & 349 & 395 & 398 & 424 \\
\hline & & $79 \%$ & $89 \%$ & $86 \%$ & $72 \%$ & $82 \%$ & $82 \%$ & $88 \%$ \\
\hline & & 8903 & 8146 & 10366 & 6850 & 11414 & 8776 & 5031 \\
\hline & & $91 \%$ & $92 \%$ & $90 \%$ & $93 \%$ & $89 \%$ & $91 \%$ & $95 \%$ \\
\hline \multirow[b]{4}{*}{$\approx$} & \multirow{4}{*}{ Step 1} & 373 & 401 & 302 & 194 & 381 & 357 & 391 \\
\hline & & $77 \%$ & $83 \%$ & $62 \%$ & $40 \%$ & $79 \%$ & $74 \%$ & $81 \%$ \\
\hline & & 13889 & 11821 & 13142 & 6845 & 11480 & 10600 & 7691 \\
\hline & & $86 \%$ & $88 \%$ & $87 \%$ & $93 \%$ & $89 \%$ & $89 \%$ & $92 \%$ \\
\hline$D$ & \multirow{4}{*}{ Step 2} & 356 & 413 & 307 & 178 & 381 & 383 & 394 \\
\hline \multirow[t]{2}{*}{$\Omega$} & & $74 \%$ & $85 \%$ & $63 \%$ & $37 \%$ & $79 \%$ & $79 \%$ & $81 \%$ \\
\hline & & 14975 & 12640 & 13478 & 6821 & 11533 & 10993 & 8454 \\
\hline $\mathbf{Z}$ & & $85 \%$ & $87 \%$ & $87 \%$ & $93 \%$ & $88 \%$ & $89 \%$ & $92 \%$ \\
\hline \multirow[t]{4}{*}{$a$} & \multirow{4}{*}{ Step 4} & 298 & 362 & 345 & 157 & 352 & 296 & 394 \\
\hline & & $62 \%$ & $75 \%$ & $71 \%$ & $32 \%$ & $73 \%$ & $61 \%$ & $81 \%$ \\
\hline & & 17828 & 13613 & 14809 & 6712 & 11544 & 11360 & 8445 \\
\hline & & $82 \%$ & $86 \%$ & $85 \%$ & $93 \%$ & $88 \%$ & $89 \%$ & $92 \%$ \\
\hline
\end{tabular}

\section{Some Practical Examples}

Theoretically, if a MB is alien and no match exists within the search window, the DPBM performs only five computations to take this decision, outperforming all other algorithms. This means that if the target frame belongs to another shot (or scene) than the reference frame (in case of abrupt shot transition), then the DPBM algorithm performs only 2420 AMDs computations to decide that the whole frame should be intra-coded, as shown in Table 2. Other algorithms will perform all possible computations to take such a decision.

Table 2. The Computations at an Abrupt Transition

\begin{tabular}{ccccccc} 
TSS & SESTSS & NTSS & 4SS & DS & ARPS & DPBM \\
\hline 25 & 25 & 33 & 27 & 27 & 12 & 5 \\
12100 & 12100 & 15972 & 13068 & 13068 & 5808 & 2420 \\
\hline
\end{tabular}

The practical results, at an abrupt shot transition achieve computation reduction of $98 \%$ that outperform all other algorithms. To be more specific, the algorithm is modified to verify the prompt termination in case of abrupt transitions by applying the histogram similarity check. The experimental results show that all abrupt transitions detected by the DPBM algorithm have been verified (an example is shown in Figure 3). 


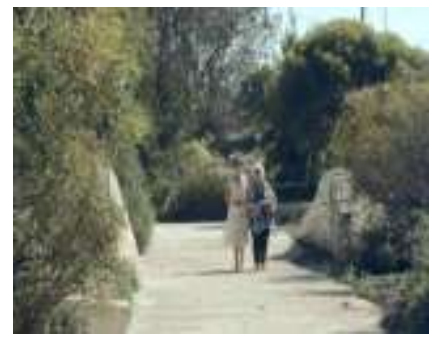

(a)

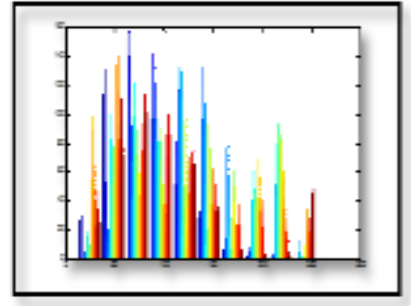

(c)

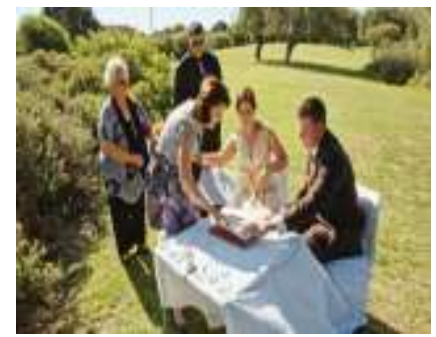

(b)

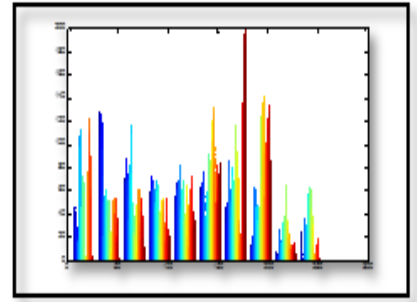

(d)

Figure 3. Two Successive Frames at Abrupt Transition in the 'Wedding' Clip: (a), (b) and their Histograms: (c), (d)

An example of the AMDs of the MBs, of the considered frame, is shown in Figure 4. There are two paths (red colored), the algorithm follow the two paths, and select the minimum as the best matched MB.

\begin{tabular}{|l|l|l|l|l|l|l|l|}
\hline 11.16 & 10.97 & 10.80 & 10.58 & 10.42 & 10.28 & 10.14 & 10.01 \\
\hline 8.13 & 7.97 & 7.79 & 7.57 & 7.40 & 7.26 & 7.13 & 7.01 \\
\hline 4.89 & 4.74 & 4.58 & 4.40 & 4.23 & 4.10 & 3.99 & 3.95 \\
\hline 4.04 & 4.10 & 4.17 & 4.23 & 4.34 & 4.47 & 4.64 & 4.86 \\
\hline 6.50 & 6.53 & 6.55 & 6.59 & 6.63 & 6.69 & 6.78 & 6.90 \\
\hline 7.32 & 7.33 & 7.34 & 7.35 & 7.34 & 7.34 & 7.36 & 7.41 \\
\hline
\end{tabular}

Figure 4. Two-Path Case of the DPBM Algorithm

The 255 AMD values of the compared MBs, a single path example for a MB in the middle of a considered frame is shown in Figure 5. The path length is 9, having $[5+(9-1) \times 3]=29$ AMD computations.

The incorrect best matched alarm shown in Figure 6, is because the best match within the search window is irrelevant, i.e., belongs to another region.

The worst case of the DPBM algorithm, exits if the trivial setup is explored at an abrupt shot transition, as shown in Figure 7. 


\begin{tabular}{|l|l|l|l|l|l|l|l|l|}
\hline 19.05 & 19.18 & 19.33 & 19.56 & 19.86 & 20.22 & 20.55 & 20.8 \\
\hline 19.29 & 19.38 & 19.52 & 19.71 & 19.98 & 20.3 & 20.67 & 20.99 \\
\hline 19.67 & 19.74 & 19.96 & 20.18 & 20.5 & 20.81 & 21.14 & 21.45 \\
\hline 20.49 & 20.53 & 20.66 & 20.87 & 21.12 & 21.41 & 21.77 & 22.14 \\
\hline 21.68 & 21.79 & 22.05 & 22.34 & 22.79 & 23.12 & 23.57 & 24.04 \\
\hline 23.43 & 23.59 & 23.86 & 24.13 & 24.52 & 24.8 & 25.14 & 25.5 \\
\hline 24.07 & 24.21 & 24.39 & 24.57 & 24.87 & 25.1 & 25.39 & 25.64 \\
\hline 23.42 & 23.52 & 23.64 & 23.75 & 24.07 & 24.3 & 24.63 & 24.95 \\
\hline 22.84 & 22.91 & 23.01 & 23.17 & 23.5 & 23.78 & 24.13 & 24.48 \\
\hline 22.11 & 22.19 & 22.34 & 22.55 & 22.89 & 23.2 & 23.58 & 23.94 \\
\hline 21.28 & 21.41 & 21.66 & 21.96 & 22.33 & 22.69 & 23.08 & 23.46 \\
\hline 20.73 & 20.97 & 21.3 & 21.68 & 22.01 & 22.36 & 22.76 & 23.16 \\
\hline 20.63 & 20.86 & 21.23 & 21.68 & 22.11 & 22.54 & 22.99 & 23.46 \\
\hline 20.92 & 21.16 & 21.51 & 21.96 & 22.39 & 22.84 & 23.27 & 23.66 \\
\hline 20.95 & 21.24 & 21.64 & 22.14 & 22.53 & 22.91 & 23.32 & 23.65 \\
\hline
\end{tabular}

Figure 6. A False Alarm by the DPBM Algorithm

\begin{tabular}{|r|r|r|r|r|r|r|}
\hline 40.816 & 41.246 & 42.043 & 42.207 & 42.637 & 44.156 & 47.645 \\
\hline 41.375 & 41.68 & 42.332 & 42.66 & 43.109 & 44.582 & 47.543 \\
\hline 41.707 & 41.984 & 42.465 & 42.926 & 43.395 & 44.855 & 47.609 \\
\hline 42.352 & 42.301 & 42.508 & 42.703 & 43.195 & 44.625 & 47.324 \\
\hline 43.27 & 42.703 & 42.543 & 42.402 & 42.711 & 44.043 & 46.684 \\
\hline 44.234 & 43.504 & 42.832 & 42.293 & 42.355 & 43.371 & 45.824 \\
\hline 45.027 & 44.367 & 43.324 & 42.348 & 42.059 & 42.637 & 44.648 \\
\hline 45.043 & 44.754 & 43.43 & 42.289 & 41.641 & 41.555 & 43.203 \\
\hline 44.445 & 44.496 & 43.344 & 42.109 & 41.148 & 40.441 & 41.43 \\
\hline 43.551 & 43.977 & 43.063 & 41.734 & 40.688 & 39.469 & 39.75 \\
\hline 42.824 & 43.09 & 42.453 & 41.355 & 40.594 & 39.008 & 38.848 \\
\hline
\end{tabular}

Figure 7. The AMDs of the Corresponding MBs at Abrupt Transition

The best case of the DPBM algorithm exists if the non-trivial setup is applied while each of the reference and target frames have similar regions, as shown in Figure 8 . 


\begin{tabular}{|r|r|r|r|r|}
\hline 14.246 & 5.5078 & 7.6523 & 10.648 & 10.629 \\
\hline 14.207 & 4.4844 & 6.4336 & 9.8633 & 9.9688 \\
\hline 14.402 & 4.5547 & 6.0664 & 9.7578 & 9.9375 \\
\hline 14.801 & 5.625 & 6.9961 & 10.309 & 10.406 \\
\hline 15.414 & 6.7383 & 7.9961 & 10.945 & 11.039 \\
\hline 15.816 & 7.9531 & 9.0156 & 11.559 & 11.719 \\
\hline
\end{tabular}

\begin{tabular}{|r|r|r|r|r|}
\hline 18.566 & 15.801 & 14.41 & 14.43 & 15.883 \\
\hline 16.258 & 11.863 & 8.5625 & 10.563 & 14.273 \\
\hline 15.148 & 8.7617 & 0.50781 & 8.0234 & 13.281 \\
\hline 15.738 & 11.004 & 8.1406 & 11.039 & 14.145 \\
\hline 17.484 & 15.168 & 13.707 & 14.566 & 16.289 \\
\hline 9.863 & 18.371 & 17.645 & 18.098 & 19.496 \\
\hline
\end{tabular}

\section{Figure 8. Samples of Direct Path to the Best Matched MB}

\section{Conclusion}

We suggested an efficient motion estimation mechanism that reduces cost in various dimensions. First, almost all the transitions separating shots and scenes in video streams are abrupt regardless of their types. Second, $45 \%$ of the inter-coded MBs are intra-coded as per reviewed in the literature. Third, the further reduction of computations is still needed to detect the best matched $\mathrm{MB}$, which contributes to improve error recovery and concealment in video coding. The proposed DPBM algorithm detects the best matched $\mathrm{MB}$ in a direct path by following the gradual decrease (degradation) of the ADM values of the corresponding MBs and their attached four neighbors.

The developed algorithm is compared to the referenced algorithms ES, TSS, NTSS, SES, 4SS, DS, and ARPS. The conducted experiments showed comparable results concerning the detection rate of the best matched MBs. On the other hand, the proposed DPBM algorithm overcomes all others concerning the number of computations in case of abrupt transitions, at which the frame will be intra-coded.

\section{References}

[1] Y.-C. Lin and S.-C. Tai, "Fast Full-Search Block-Matching Algorithm for Motion-Compensated Video Compression", IEEE Transactions on Communications, vol. 45, no. 5, (1997).

[2] A. Barjatya, "Block matching algorithms for motion estimation", IEEE Transactions Evolution Computation, vol. 8, no. 3, (2004), pp. 225-239

[3] 3.R. Yaakob, A. Aryanfar, A. Abdul Halin and N. Sulaiman, "A Comparison of Different Block Matching Algorithms for Motion Estimation" The $4^{\text {th }}$ International Conference on Electrical Engineering and Informatics ICEEI, (2013).

[4] H. Kalva and B. Furht, "Complexity Estimation of the H.264 Coded Video Bitstreams", The Computer Journal (C) Oxford Univ. Press, vol. 48, no. 5, (2005).

[5] B. Damerchilu, M. Reza Meybodi and M. Sadegh Norouzzadeh, "Motion Estimation Using Learning Automata", Machine Vision and Applications archive, Springer-Verlag, vol. 27, iss. 7, (2016), pp. 10471061.

[6] H.-W. Cheng and L.-R. Dung, "EFBLA: a two-phase matching algorithm for fast motion estimation", Electronics Letters, vol. 40, no. 11, (2004).

[7] H. Li, Z. G. Li and C. Wen, "Fast Mode Decision Algorithm for Inter-Frame Coding in Fully Scalable Video Coding”, IEEE Transactions on Circuits and Systems for Video Technology, vol. 16, no. 7, (2006).

[8] H. L. Oh, Y. Wai and L. Hui, “Adaptive Motion Estimator”, US patent no. US 7,551,673 B1, (2009). 


\section{Authors}

Mona A. M. Fouad, she is currently working at the National Telecommunication Institute of Egypt (NTI). She had her PhD in 2008 from Cairo University; she led many projects concerning e-learning, digital image/video processing and content analysis. She published many papers in national and international journals and conferences. She is teaching image processing and multimedia Communications for post-graduate level at NTI. Currently, Dr. Fouad practices researches in the domain of content analysis over large scale networks.

Ahmed Mokhtar A. Mansour, he works as CTO for the Nile Innovations Company in Egypt and he also provides independent consultancy for ITIDA, TIEC, MCIT, Cairo Orange Lab, Qatar foundation, Saudi ARAMCO, Central Bank of Morocco. Ahmed received his $\mathrm{PhD}$ in 2003 from IRISA-Rennes1 University in France. He teaches at many universities in Egypt and in the United Arab Emirates. Dr. Mokhtar participated in many EU projects and lead efforts in many of them. He was the head of open-innovation at Cairo Orange Lab and the regional manager of VAS researches for AMEA region. Dr. Mokhtar research domains are: computer networks, RFID applications, QoS of online interactive services over hybrid networks using CDN, and VAS for mobile systems. 